\title{
Validación del IPP-R con alumnos de cuarto y quinto grado de secundaria de colegios públicos y privados de Lima metropolitana
}

\author{
Cipriano Olivera \\ Patricia Uribe \\ Nadia Denegri \\ Universidad de Lima
}

Recibido: 14 de febrero del 2013 / Aprobado: 4 de julio del 2013

El inventario de intereses y preferencias profesionales revisado IPP-R (2010) se estandarizó y validó en Lima metropolitana, tomando como referencia los estudios sobre desarrollo humano que realiza la Unesco en el Perú y cuyos datos se refieren al año 2007. La muestra fue de 1567 alumnos de ambos sexos, del cuarto y quinto grado de secundaria de colegios públicos y privados. Se efectuaron cálculos descriptivos en función de las características de la muestra. Igualmente, a nivel de inferencia, se calculó la consistencia interna sobre la base de las pruebas del alfa de Cronbach, la validez de constructo empleando el KMO y la prueba de esfericidad de Bartlett, así como las correlaciones item-test y el análisis factorial.

intereses y preferencias / campos profesionales / validez de constructo / análisis factorial

Validation of the IPP-R on $4^{\text {th }}$ and $5^{\text {th }}$ secondary students of public and private schools in metropolitan Lima

The purpose of this research project was to standardize and validate the Revised Professional Interests and Preferences Inventory (IPP-R 2010) in metropolitan Lima on the basis of studies on human development data provided by Unesco in 2007. The population sample consisted of 1567 female and male students attending 4th and 5th year of secondary education in private and public schools. We carried out a descriptive calculus based on the characteristics of the sample, calculated internal consistency based on Cronbach Alpha score, Construct Validity through $K M O$, the significance value of Bartlett esfericity test, item-test correlations and factor analysis.

interests and preferences / professional fields / construct validity / factor analysis

Correo electrónico: colivera@ulima.edu.pe 


\section{INTRODUCCIÓN}

La métrica en Psicología señala la importancia y la necesidad de que los instrumentos de evaluación que se utilizan sean válidos y confiables. Dicha condición es entendida y aceptada por cuanto los profesionales del área toman decisiones sobre personas que se sustentan muchas veces en resultados obtenidos a través de pruebas. La pregunta que se desprende de la presente idea es: ¿qué sucede si se utiliza un instrumento de otra realidad sociocultural a la nuestra?, ¿hasta qué punto los baremos utilizados para interpretar los resultados son los adecuados?, ¿de qué manera las personas pueden verse afectadas por un diagnóstico que tiene un sesgo de origen? La respuesta a estas interrogantes solo tiene una solución: crear pruebas psicológicas que respondan a realidades específicas o adaptar las existentes para que resulten funcionales en un contexto determinado.

El presente trabajo de investigación se inscribe dentro del marco de las soluciones y por ello se propuso validar el Inventario de Preferencias Profesionales (IPP-R) en alumnos del cuarto y quinto grado de secundaria de colegios públicos y privados de Lima metropolitana, previo proceso de adaptación.

\section{ADAPTACIÓN DE INSTRUMENTOS DE} MEDICIÓN

Los test, en cualquiera de sus modalidades, constituyen los instrumentos de medición más utilizados por los psicólogos en sus diferentes campos de acción. Su utilización no solo requiere la experticia de los profesionales que los aplican, también la formalidad en cuanto a su construcción y la necesidad de tomar en cuenta el contexto en el cual son administrados (Muñiz \& Hambleton, 1996).

Como es de suponer, el proceso de globalización que ha alcanzado a diferentes campos del conocimiento humano, sin dejar de lado el de las pruebas psicológicas, conduce a que, cada vez con mayor rapidez, un test creado en una realidad determinada se conozca y se utilice en una realidad distinta. Esta situación plantea un tema al que se debe prestar especial atención: los test no son instrumentos automáticamente utilizables, deben adaptarse en función de diferencias interculturales.

Frente a esta necesidad, la Comisión Internacional de Test, ITC (por sus siglas en inglés), ha propuesto una serie de recomendaciones que pretenden sistematizar el proceso de adaptación y validación de instrumentos de medición.

La ITC ha llamado la atención acerca de posibles fuentes de error en los que se puede incurrir cuando se pretende adaptar un test de una cultura a otra. De allí que se ha interesado por proponer una serie de directrices en torno a los siguientes aspectos:

- El contexto.- En este aspecto las directrices tratan de asegurar que haya una equivalencia de los constructos 
medidos en las dos poblaciones de interés. A medida que la distancia cultural entre dos o más poblaciones de interés aumenta, existen más probabilidades de que haya diferencias entre los constructos.

- La construcción y adaptación.- Se logra cuando los constructores del test han aportado los datos que garantizan que hay una equivalencia apropiada entre los constructos a medir en ambas poblaciones. Otro aspecto clave subrayado en varias de las directrices es que los constructores recojan datos de todo tipo que permitan evaluar la validez y comparar las dos versiones.

- La aplicación.- Las directrices, en este sentido, subrayan aquellos aspectos claves para una aplicación correcta de los test, ya que algunas veces se descuida confiándola a personas sin la debida formación.

- Interpretación de las puntuaciones.Es importante tomar en cuenta que las puntuaciones de los test siempre son datos que deben de utilizarse convergentemente con otros en la toma de decisiones.

\section{LA ORIENTACIÓN VOCACIONAL}

La dimensión aplicada de la psicología pretende el beneficio de las personas y las organizaciones en sus más variadas circunstancias individuales y sociales. En este contexto, un aspecto complejo del comportamiento de socialización es la conducta vocacional.

Rivas (1995) señala que la libertad de elegir implica riesgos y oportunidades que están disponibles para el sujeto a lo largo de su desarrollo. En este proceso se pueden producir situaciones donde las personas, en función de su maduración personal, de la trascendencia de las opciones a elegir y de la riqueza o limitaciones del entorno, necesiten algún tipo de ayuda para intentar resolver el problema de relacionarse con el mundo productivo de los adultos.

A decir de Gavilán (2006), pensar en los orígenes de la orientación profesional implica remontarse a los inicios de la historia de la humanidad. En este sentido, a pesar de los diferentes cuadros interpretativos, la mayoría de los investigadores reconoce dos etapas en el plano de la orientación: una precientífica y otra formal o científica.

Crites (1969) clasifica las teorías relacionadas con la orientación vocacional de la siguiente manera:

- Teorías no psicológicas.- En este contexto, la elección vocacional estaría influenciada por situaciones externas al individuo. Su personalidad, intereses y motivaciones no constituirían elementos mediadores en este sentido.

- Teorías psicológicas.- En este grupo destacan propuestas que priorizan los rasgos personales, los intereses 
y las aptitudes, así como propuestas motivacionales, evolutivas y vinculadas con la toma de decisiones.

- Teorías generales o integrales.- Este grupo de teorías analizan las elecciones vocacionales buscando la interacción de distintas disciplinas. Destacan las propuestas relacionadas con el aprendizaje social, la relación personal-ambiente y los enfoques multiculturales.

\section{LOS INTERESES VOCACIONALES}

Hablar de intereses en el contexto vocacional es ocuparse de uno de los tópicos clásicos y con mayor frecuencia utilizados en psicología en esta área.

No existe una única definición de este concepto y cada una de ellas enfoca un aspecto que lo caracteriza. De allí, tal como señala Super (1967), «interés» es una palabra que designa una serie de intenciones y concluye que existen tres hipótesis de trabajo que orientan el tratamiento de los intereses en el campo de la orientación vocacional:

- No existe diferencia de naturaleza entre intereses y aptitudes.

- El interés es un aspecto de la personalidad.

- El interés no es una aptitud ni un rasgo de la personalidad, sino algo distinto e irreductible.

Rivas (1994) señala que, a pesar de las diferentes concepciones que existen en torno a los intereses, estas coinciden en otorgarle un carácter motivador y reforzante, calificándolo como el motor de la conducta vocacional. Un acuerdo bastante amplio se produce también a la hora de considerar que el interés se aprende, fundamentalmente, en interacción; por lo tanto, hay que recordar que el interés vocacional de los individuos evoluciona, mostrándose poco consistente hasta la juventud (Rocabert, 1987, en Rivas, 1994).

Tomando en cuenta las diferentes propuestas relacionadas con los intereses, estos pueden definirse como tendencias o preferencias por escoger, entre ciertas ocupaciones o profesiones, por encontrar en ellas satisfacción, como producto de sus aptitudes y formas de comportamiento condicionadas por las experiencias y posibilidades ofrecidas por el medioambiente (Hernández, 2001).

A partir de las definiciones de los diferentes autores pueden considerarse tres factores como los componentes de los intereses:

- Cognitivo, por su relación con la atención.

- Afectivo, relacionado con la sensación de placer o agrado.

- Conductual, relacionado con la tendencia a «actuar hacia» o reaccionar positivamente (Contreras, 2000).

Montero (2005) señala que en el estudio de la relación entre intereses y la elección de la carrera, algunos teóricos 
han planteado que dicho proceso de decisión es una síntesis compleja de muchos elementos, aparte de los intereses, lo que ha llevado a clasificarlos de la siguiente manera:

- Intereses expresados.- Consisten en la confesión verbal de los intereses por un objeto, actividad o profesión. Los intereses expresados por los niños y adolescentes son inestables, varían según la madurez y experiencia del individuo. En algunos casos, estos intereses representan «fantasías o caprichos temporales» y no proporcionan datos útiles para el diagnóstico o el pronóstico.

- Intereses manifiestos.- Son aquellos intereses que se observan en las acciones de las personas y en su participación en diversas actividades.

- Intereses inventariados.- Son aquellos intereses estimados a través de las respuestas hechas a una lista de preguntas sobre gustos y aversiones, también por el orden asignado a una serie de actividades en razón de la preferencia que siente por ellos. Estas respuestas se evalúan de acuerdo a normas obtenidas mediante procedimientos estadísticos.

- Intereses vocacionales.- De acuerdo con Holland (1965), los intereses vocacionales se construyen como una expresión de la personalidad; entonces, representan la expresión de la personalidad en el trabajo, en las materias escolares, en los pasatiem- pos, en las actividades recreativas y en las preferencias profesionales.

Asimismo, Cortada (1977) señala que un interés vocacional nace de una tendencia que se produce espontáneamente y cuyas motivaciones son a menudo muy profundas y dirigen al individuo en cierto sentido, que se expresa por una concentración de un esfuerzo de tipo duradero hacia el ejercicio de actividades profesionales.

La estabilidad de los intereses vocacionales aumenta a partir de los 17 años en el periodo aproximado en que se inicia la «educación superior», observando que la estabilidad de estos se encuentra en relación directa con la edad y el grado escolar. Se puede entonces afirmar dentro de este marco, que los intereses no maduros de la niñez se modifican en la adolescencia, encaminándose hacia actividades más reales y concordantes con sus verdaderas aptitudes, sin llegar a la plena madurez hasta el principio de la vida adulta (Strong, 1993, en Anastasi, 1998).

\section{ObJeTIVOS DE LA INVESTIGACIÓN}

1. Adaptar el inventario de intereses y preferencias profesionales revisado (IPP-R) en alumnos del cuarto y quinto grados de educación secundaria de instituciones educativas públicas y privadas de Lima metropolitana.

2. Validar el inventario de intereses y preferencias profesionales revisado 
(IPP-R) para alumnos del cuarto y quinto año de educación secundaria de instituciones educativas públicas y privadas de Lima metropolitana.

3. Determinar los índices de confiabilidad del inventario de intereses y preferencias profesionales revisado (IPP-R) para alumnos del cuarto y quinto grados de educación secundaria de instituciones educativas públicas y privadas de Lima metropolitana.

4. Elaborar baremos en función del género, grado de estudio y tipo de gestión educativa.

5. Establecer las semejanzas y diferencias en intereses y preferencias profesionales en los alumnos según las variables controladas.

6. Elaborar un módulo psicométrico de aplicación en el campo de la psicología educativa con relación al constructo intereses y preferencias profesionales.

\section{Metodología}

\section{Participantes}

Para efectos de la investigación constituyeron sujetos de estudio alumnos del cuarto y quinto grado de secundaria de instituciones educativas públicas y privadas de Lima metropolitana. Los estudios normativos y de adaptación se caracterizan por presentar muestras suficientemente grandes. En este sentido, para determinar el tamaño de la mues- tra asumimos la propuesta de Nunnally \& Bernstein (1995), quienes plantean que esta debe incluir hasta 10 veces el número de participantes con relación al número de ítems del instrumento. Esto nos llevó a considerar 1800 sujetos, tomando en cuenta que la cantidad de ítems del instrumento a validar ascendía a 180 .

El procedimiento de la selección de la muestra se realizó considerando una preselección de los distritos de Lima metropolitana agrupados en cuatro bloques de mayor a menor nivel de desarrollo humano, según datos de las investigaciones de la Unesco en el Perú en el 2007. De esta manera, se identificaron cinco distritos por cada bloque de desarrollo y, de cada uno de ellos, se seleccionó un colegio particular y uno estatal, lo que hacía un total de 10 colegios por bloque y un total de 40 colegios (20 estatales y 20 particulares), de los cuales finalmente se extrajo la muestra.

La edad al momento de levantar la encuesta fue de 15,8 años en promedio, con una dispersión de 0,81 años, en un rango que va de los 14 a los 20 años.

La distribución de la muestra según sexo fue proporcional, $50 \%$ de sexo masculino y $50 \%$ de sexo femenino.

Asimismo, 49,65\% de los alumnos de quinto grado era de sexo masculino, mientras que el 50,35\% eran mujeres; se puede afirmar que existe una distribución homogénea para estas dos categorías. Además, se puede observar que la distribución para cuarto grado es de 
Tabla 1

Distribución por grado y sexo

\begin{tabular}{lccccccc}
\hline & \multicolumn{5}{c}{ Grado } & \multicolumn{2}{c}{} \\
\cline { 2 - 6 } Sexo & \multicolumn{2}{c}{$\begin{array}{c}\text { Quinto } \\
(\mathrm{n}=725)\end{array}$} & \multicolumn{2}{c}{$\begin{array}{c}\text { Cuarto } \\
(\mathrm{n}=\mathbf{8 4 2})\end{array}$} & \multicolumn{2}{c}{ Total } \\
\cline { 2 - 6 } & $f$ & $\%$ & $f$ & $\%$ & $f$ & $\%$ \\
\hline Masculino & 360 & 49,65 & 423 & 50,23 & 783 & 49,96 \\
Femenino & 365 & 50,35 & 419 & 49,77 & 784 & 50,04 \\
\hline
\end{tabular}

$50,23 \%$ para el sexo masculino y $49,77 \%$ para el sexo femenino, igualmente homogéneas para dichas categorías.

Según grado y edad, 725 alumnos pertenecen al quinto grado, que representa un $46,27 \%$, y 842 al cuarto gra- do, que constituye el $53,73 \%$. De toda la muestra estudiada, la mayor concentración porcentual se encuentra entre los 15-17 años, con el 81,18 \% de concentración.

Tabla 2

Distribución por grado y edad

\begin{tabular}{rrrrrrr}
\hline Edad & \multicolumn{2}{c}{ Quinto grado } & \multicolumn{2}{c}{ Cuarto grado } & \multicolumn{2}{c}{ Total } \\
\hline & \multicolumn{1}{c}{$\boldsymbol{f}$} & \multicolumn{1}{c}{$\%$} & \multicolumn{1}{c}{$\boldsymbol{f}$} & $\%$ & \multicolumn{1}{c}{$\boldsymbol{f}$} & $\%$ \\
\hline 14 & 0 & 0 & 37 & 4,39 & 37 & 2,37 \\
15 & 44 & 6,07 & 500 & 59,39 & 544 & 34,72 \\
16 & 470 & 64,83 & 258 & 30,65 & 728 & 46,46 \\
17 & 176 & 24,28 & 42 & 4,99 & 218 & 13,91 \\
18 & 34 & 4,68 & 3 &, 36 & 37 & 2,36 \\
19 & 1 &, 13 & 1 &, 11 & 2 &, 12 \\
20 & 0 & 0 & 1 &, 11 & 1 &, 06 \\
Total & 725 & 46,27 & 842 & 53,73 & 1567 & 100,00 \\
\hline
\end{tabular}




\section{DESCRIPCIÓN DEL INSTRUMENTO}

Con fines de adaptación y validación se utilizó el inventario de intereses y preferencias profesionales revisado (IPP-R), elaborado por María Victoria de la Cruz López (2010). Previamente se consultó y solicitó la correspondiente autorización a los editores para el uso del instrumento con fines de investigación. A continuación se indica la ficha técnica:
La primera fase del trabajo consistió en realizar la adaptación del inventario a través de la revisión y análisis de los ítems de la prueba por parte del equipo de investigación en función de las características de los estudiantes. En esta primera fase se realizaron modificaciones a 127 ítems y solo 53 mantuvieron su estructura original, lo cual ya significaba un cambio significativo. Se mantuvo la intencionalidad de cada ítem al

\section{Ficha técnica}

Nombre:

Autor:

Adaptado en Lima por:

Aplicación:

Ámbito de aplicación:

Duración de la aplicación:

Campo de intereses:

Calificación:

Finalidad:

Baremación:
Inventario de preferencias profesionales IPP-R

María Victoria de la Cruz (TEA, 2010)

Cipriano Olivera Guzmán, Patricia Uribe Ybáñez y Nadia Denegri Orderique

Individual y colectiva

Alumnos del $4^{\circ}$ y $5^{\circ}$ grado de educación secundaria.

30 y 45 minutos.

El instrumento mide quince campos de interés: científico, técnico, sanitario, científico social-humanidades, jurídico-social, comunicación-información, psicopedagógico, empresarialadministrativo-comercial, informática, agrario-agropecuarioambiental, artístico plástico-artesanía-moda, artístico musicalespectáculo, fuerzas armadas-seguridad-protección, deportivo y turismo y hotelería

Cada uno de los 180 ítems de que consta la prueba puede alcanzar tres tipos de puntaje:

No me gusta o no conozco: $\quad 0$

Me es indiferente o Tengo dudas: 1

Me gusta:

Apreciación de los intereses de los alumnos con relación a 15 campos profesionales, teniendo en cuenta la información sobre las profesiones y tareas que involucran.

Percentiles escolares realizados con muestras de alumnos del $4^{\circ}$ y $5^{\circ}$ de secundaria. 
no realizar cambios que desvirtuaran dicho propósito.

Esta primera versión de la prueba adaptada fue sometida a la validación por criterio de jueces, habiendo recibido el aporte de profesionales pertenecientes a la Universidad Ricardo Palma, la Universidad de Lima y la Universidad Femenina del Sagrado Corazón. Se obtuvo la $\mathrm{V}$ de Aiken con un valor de 0.87 , lo que validó el contenido de los ítems.

La nueva propuesta de inventario, con las modificaciones efectuadas, fue sometida a una prueba piloto en cuatro centros educativos (dos estatales y dos particulares) con un total de 173 alumnos evaluados, los cuales no fueron considerados en la muestra final.
Entre los resultados de la versión piloto se puede señalar que se halló una confiabilidad mediante el alfa de Cronbach, con índices considerados elevados: 0.965 para la prueba total, de 0.96 en la muestra de instituciones de gestión estatal y 0.95 en instituciones de gestión particular. Igualmente, índices de 0.96 en la muestra de cuarto grado y 0.97 en la de quinto grado. Solo se encontraron varianzas pequeñas en los ítems $16,66,68,114$ y 158 , pero que al ser retirados del análisis, el alfa de Cronbach no se incrementaba en ningún caso, por lo cual se decidió considerarlos como parte de la prueba.

El número de ítems y puntajes por cada componente de las áreas ocupacionales se puede observar en la siguiente tabla:

Tabla 3

Distribución de ítems y puntajes según componentes por cada grupo ocupacional

\begin{tabular}{lccccccc}
\hline & \multicolumn{2}{c}{ C. profesión } & \multicolumn{2}{c}{ C. actividad } & \multicolumn{2}{c}{ Total } \\
\cline { 2 - 7 } Área & $\begin{array}{l}\mathbf{N}^{\mathbf{0}} \text { de } \\
\text { ítems }\end{array}$ & $\begin{array}{l}\text { Puntaje } \\
\text { máximo }\end{array}$ & $\begin{array}{l}\mathbf{N}^{\mathbf{0}} \text { de } \\
\text { ítems }\end{array}$ & $\begin{array}{l}\text { Puntaje } \\
\text { máximo }\end{array}$ & $\begin{array}{l}\mathbf{N}^{\circ} \text { de } \\
\text { Ítems }\end{array}$ & $\begin{array}{l}\text { Puntaje } \\
\text { máximo }\end{array}$ \\
\hline $\begin{array}{l}\text { 1. Científico } \\
\text { 2. Técnico }\end{array}$ & 7 & 14 & 8 & 16 & 15 & 30 \\
$\begin{array}{l}\text { 3. Sanitario } \\
\text { 4. Científico social- }\end{array}$ & 5 & 10 & 6 & 12 & 11 & 22 \\
humanidades & 5 & 10 & 5 & 10 & 10 & 20 \\
$\begin{array}{l}\text { 5. Jurídico-social } \\
\text { 6. Comunicación- }\end{array}$ & 4 & 10 & 5 & 10 & 10 & 20 \\
$\quad$ información & 7 & 14 & 7 & 14 & 14 & 28 \\
\hline
\end{tabular}




\begin{tabular}{|c|c|c|c|c|c|c|}
\hline \multirow{2}{*}{ Área } & \multicolumn{2}{|c|}{ C. profesión } & \multicolumn{2}{|c|}{ C. actividad } & \multicolumn{2}{|c|}{ Total } \\
\hline & $\begin{array}{l}\mathrm{N}^{\circ} \mathrm{de} \\
\text { ítems }\end{array}$ & $\begin{array}{l}\text { Puntaje } \\
\text { máximo }\end{array}$ & $\begin{array}{l}\mathrm{N}^{\circ} \mathrm{de} \\
\text { ítems }\end{array}$ & $\begin{array}{l}\text { Puntaje } \\
\text { máximo }\end{array}$ & $\begin{array}{l}\mathrm{N}^{\circ} \mathrm{de} \\
\text { Ítems }\end{array}$ & $\begin{array}{l}\text { Puntaje } \\
\text { máximo }\end{array}$ \\
\hline 7. Psicopedagógico & 4 & 8 & 6 & 12 & 10 & 20 \\
\hline $\begin{array}{l}\text { 8. Empresarial } \\
\text { administrativo- } \\
\text { comercial }\end{array}$ & 5 & 10 & 6 & 12 & 11 & 22 \\
\hline 9. Informática & 5 & 10 & 7 & 14 & 12 & 24 \\
\hline $\begin{array}{l}\text { 10. Agrario- } \\
\text { agropecuario- } \\
\text { ambiental }\end{array}$ & 5 & 10 & 6 & 12 & 11 & 22 \\
\hline $\begin{array}{l}\text { 11. Artístico plástico- } \\
\text { artesanía- modas }\end{array}$ & 7 & 14 & 8 & 16 & 15 & 30 \\
\hline $\begin{array}{l}\text { 12. Artístico-musical- } \\
\text { espectáculo }\end{array}$ & 6 & 12 & 6 & 12 & 12 & 24 \\
\hline $\begin{array}{l}\text { 13. Fuerzas armadas- } \\
\text { seguridad- } \\
\text { protección }\end{array}$ & 7 & 14 & 7 & 14 & 14 & 28 \\
\hline 14. Deportivo & 5 & 10 & 8 & 16 & 13 & 26 \\
\hline $\begin{array}{l}\text { 15. Turismo y } \\
\text { hotelería }\end{array}$ & 4 & 8 & 8 & 16 & 12 & 24 \\
\hline
\end{tabular}

En la versión original del manual realizada en España, la tipificación del IPP-R se llevó a cabo con 1256 sujetos que cursaban el segundo y cuarto grado de educación secundaria obligatoria y sujetos de primer y segundo año de ba- chillerato. De estos sujetos 633 fueron varones y 623 mujeres.

Para el estudio de la fiabilidad se trabajó con el alfa de Cronbach, para cada una de las escalas de manera independiente, tal como se muestra en la tabla 4 : 
Tabla 4

Coeficientes alfa de Cronbach para el total de cada escala de la versión original

\begin{tabular}{lc}
\hline Escala & Alfa de Cronbach \\
\hline Científico & 0.90 \\
Técnico & 0.90 \\
Sanitario & 0.92 \\
Científico social-humanidades & 0.81 \\
Jurídico-social & 0.86 \\
Comunicación-información & 0.89 \\
Psicopedagógico & 0.90 \\
Empresarial administración-comercial & 0.90 \\
Informática & 0.96 \\
Agrario-agropecuario-ambiental & 0.86 \\
Artístico-plástico-artesanía-moda & 0.87 \\
Artístico-musical-espectáculo & 0.88 \\
Fuerzas armadas-seguridad-protección & 0.91 \\
Deportivo & 0.92 \\
Turismo y hotelería & 0.86 \\
\hline
\end{tabular}

\section{CONFIABILIDAD}

El alfa de Cronbach es un coeficiente que sirve para medir la fiabilidad de una escala de medida. Para la interpretación es suficiente que se aproxime a su valor máximo 1, para afirmar que existe mayor fiabilidad de la escala. Además, en determinados contextos, se considera que valores del alfa superiores a $0.70 \mathrm{o}$ 0.80 son suficientes para garantizar la confiabilidad de la escala.

Los criterios para el análisis utilizado en la interpretación del coeficiente alfa de Cronbach son los valores propuestos por Dorrego (1981, en Pacheco, 2008, pp. 23-24), los cuales se detallan a continuación:
Tabla 5

Valores para la interpretación del alfa de Cronbach

\begin{tabular}{cc}
\hline Valores & $\begin{array}{c}\text { Criterio de } \\
\text { confiabilidad }\end{array}$ \\
\hline $0.00-0.20$ & Ligera \\
$0.21-0.40$ & Baja \\
$0.41-0.70$ & Moderada \\
$0.71-0.90$ & Alta \\
$0.91-1.00$ & Muy alta \\
\hline
\end{tabular}

Tomando en cuenta los criterios de confiabilidad, se puede observar en la tabla 6 que todos los estadísticos de fiabilidad (alfa de Cronbach) están por encima de 0.55 , variando de moderada a alta confiabilidad. 
Por otro lado, en cinco de los campos profesionales el alfa de Cronbach del área profesión es superior al de actividad, siendo dichos campos los siguientes: empresarial-administración-comercial, informática, artístico plástico-artesaníamoda, fuerzas armadas-seguridad-protección y deportivo; en los demás cam- pos, el alfa de Cronbach es más alto en el área de actividad.

Por otro lado, los valores alfa más elevados corresponden al campo deportivo con .83 (profesión) y científico con .82 (actividad), los valores más bajos corresponden al campo jurídico-social tanto en profesión (.52), como en acti$\operatorname{vidad}(.57)$.

Tabla 6

Distribución valores alfa de Cronbach para determinar la confiabilidad de los componentes profesión y actividad

\begin{tabular}{lcc}
\hline \multirow{2}{*}{ Áreas } & Profesión & Actividad \\
\cline { 2 - 3 } & Alfa de Cronbach & Alfa de Cronbach \\
\hline Científico & .79 & .82 \\
Técnico & .63 & .73 \\
Sanitario & .73 & .73 \\
Científico social-humanidades & .63 & .63 \\
Jurídico-social & .52 & .57 \\
Comunicación-información & .70 & .75 \\
Psicopedagógico & .71 & .75 \\
Empresarial-administración-comercial & .74 & .70 \\
Informática & .77 & .71 \\
Agrario-agropecuario-ambiental & .64 & .76 \\
Artístico plástico-artesanía-moda & .75 & .73 \\
Artístico musical-espectáculo & .76 & .76 \\
Fuerzas armadas-seguridad-protección & .79 & .73 \\
Deportivo & .83 & .75 \\
Turismo-hotelería & .68 & .78 \\
\hline
\end{tabular}




\section{VALIDEZ}

Para determinar la validez de constructo se procedió a calcular el análisis factorial con la finalidad de identificar el número mínimo de factores necesarios para explicar las intercorrelaciones entre los ítems que conforman cada una de las áreas (profesión y actividad) de los diferentes campos profesionales.

En la tabla 7 se observan los indicadores fundamentales para decidir el modelo factorial adecuado, para ello analizaremos las primeras columnas donde se encuentra el KMO y la prueba de esfericidad de Bartlett. Con la prueba KMO verificamos la bondad del estadístico obtenido, que oscila entre $0 \mathrm{y}$ 1 ; cuanto más se aproxime a 1 el procedimiento es adecuado, pero si el valor es pequeño, cuanto más se aproxime a cero, el procedimiento será inadecuado. Para nuestro caso todos los KMO oscilan entre 0.61 y 0.88 ; por lo tanto, las correlaciones parciales son grandes y el procedimiento es adecuado.

En el caso del área profesión, el valor más alto corresponde al campo científico con un KMO de .85 (alta) y el más bajo al campo turismo-hotelería con .61 (moderada); en lo que respecta al área actividad el valor KMO más alto corresponde al campo científico con .88 (alta) y el valor más bajo al campo jurídico-social con .66 (moderada).

La prueba de Bartlett contrasta si la matriz de correlaciones es una matriz de identidad como hipótesis nula, es decir que las variables no están correlacionadas, en caso de aceptarse esta hipótesis indicaría que el modelo factorial es inadecuado. Para nuestro caso, en todos los campos profesionales, tanto para las profesiones como para las actividades, se rechazan las hipótesis nulas, concluyéndose que las variables están correlacionadas y confirmándose que los modelos factoriales son adecuados.

En la misma tabla 7 se observa la varianza total explicada, el número de factores y el autovalor. Para decidir el número de factores que se deben extraer utilizamos el porcentaje de la varianza total de los datos, de tal manera que el porcentaje de varianza debe explicar un valor que consideremos válido; para el caso de las ciencias sociales, es normal considerar sobre el $40 \%$ de la varianza total.

Para el caso de nuestra investigación, observamos que los autovalores en la mayoría de los casos están definidos en un solo factor, con excepción de cuatro campos: técnica (área actividad), comunicación-información (área profesión), agrario-agropecuario-ambiental (área profesión) y fuerzas armadas-seguridadprotección (área actividad) que están definidas en dos factores.

En cuanto a la mayor varianza total explicada, en el área profesión el campo deportivo muestra la mayor varianza total explicada y definida en un solo 


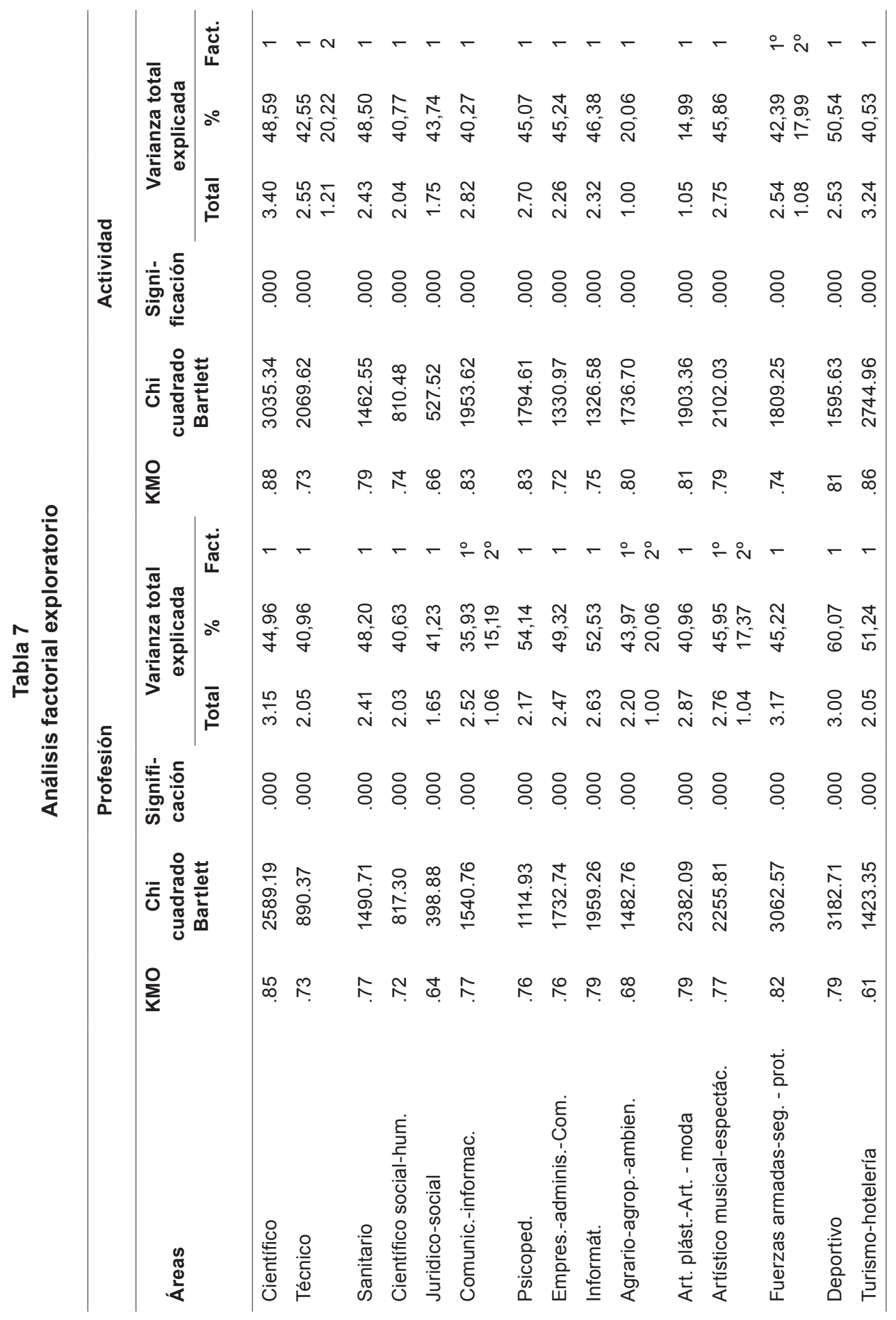


factor que se traduce en el porcentaje más elevado de la varianza total con $60 \%$; en cuanto a los campos con el porcentaje más bajo de la varianza total explicada lo constituyen el artísticoplástico-artesanía y el científico-socialhumanidades con $40 \%$. En cuanto al área actividad, el campo deportivo definido en un solo factor presenta el porcentaje más elevado con un $50 \%$, que explica la varianza total; el campo que presenta el porcentaje más bajo y que explica la varianza total es el artísticoplástico-artesanía con 14,99\%.

\section{CoMPARACIÓN DE LOS RESULTADOS}

En estadística la prueba U de Whitney, también llamada de Mann-WhitneyWilcoxon, prueba de suma de rangos de Wilcoxon o prueba de Wilcoxon-MannWhitney, es una prueba no paramétrica con la cual se identifican diferencias entre dos poblaciones basadas en el análisis de dos muestras independientes, cuyos datos han sido medidos al menos en una escala de nivel ordinal.

En las tablas 8 y 9 se observa el puntaje alcanzado en los diferentes campos profesionales tanto en las áreas profesión como actividad, estableciendo la comparación según los variables género, gestión y grado; se asume que si la significación (p) obtenida es menor de .05 se rechaza la hipótesis nula y se concluye que existe diferencia estadística significativa.

\section{Área profesión}

\section{Género}

En la tabla 8 podemos señalar que los valores $\mathrm{p}$ van desde un ,000 (técnico, sanitario, psicopedagogo, informática, artístico plástico-artesanía-moda, artístico-musical- espectáculo, fuerzas armadas-seguridad-protección, deportivo y turismo-hotelería) hasta un .419 (científico social-humanidades). En general, se han encontrado diferencias significativas por cuestión de género en 12 de los 15 campos profesionales evaluados.

\section{Gestión}

En lo que respecta a la variable gestión (particular-estatal) también se encontraron diferencias significativas en 12 de los 15 campos evaluados, oscilando sus valores desde un $p=.000$ (científico social-humanidades y fuerzas armadasseguridad-protección) hasta un $\mathrm{p}=.046$ (comunicación-información).

\section{Grado de estudio}

Finalmente, es en la variable grado de estudio donde la relación de significación es inversamente proporcional, dado que solo se han encontrado diferencias significativas en tres de los quince grupos evaluados, siendo sus intereses indistintos a que sean de cuarto o quinto de secundaria; los campos 
profesionales donde se obtuvieron diferencias significativas fueron: técnico $(p=.030)$, sanitario $(p=.030)$ y artístico plástico-artesanía y moda $(p=.020)$. En general, los valores oscilan desde un $\mathrm{p}=$ .020 hasta un $\mathrm{p}=.978$ y se pueden visualizar en la tabla 8 .

\section{Área actividad}

\section{Género}

Tratándose del componente actividad y según los datos de la tabla 9 , se puede ratificar la trascendencia de la varia- ble género en cuanto al interés de los jóvenes por una serie de actividades relacionadas con ciertos campos profesionales que están determinadas por su condición de varón o mujer, y que se ha hallado en 14 de los 15 campos profesionales con valores significativos $\mathrm{p}<$ .05 con excepción del área científico, que obtiene un $\mathrm{p}=.959$.

\section{Gestión}

En la presente variable se observa la misma tendencia que en el caso de la

Tabla 8

Comparación de la profesión según género, gestión y grado

\begin{tabular}{lcccccc}
\hline & \multicolumn{5}{c}{ Profesión } \\
\cline { 2 - 7 } Áreas & \multicolumn{2}{c}{ Género } & \multicolumn{2}{c}{ Gestión } & Grado \\
\cline { 2 - 7 } & $\mathbf{Z}$ & $\mathbf{p}$ & $\mathbf{Z}$ & $\mathbf{p}$ & $\mathbf{Z}$ & $\mathbf{p}$ \\
\hline 1. Científico & -2.19 & .029 & -2.14 & .032 & -1.81 & .070 \\
2. Técnico & -13.27 & .000 & -2.65 & .008 & -2.17 & .030 \\
3. Sanitario & -7.72 & .000 & -2.65 & .008 & -2.17 & .030 \\
4. Científico social-humanidades & -0.81 & .419 & -3.63 & .000 & -0.33 & .744 \\
5. Jurídico-social & -2.62 & .009 & -1.14 & .253 & -0.60 & .548 \\
6. Comunicación-información & -3.04 & .002 & -1.99 & .046 & -0.14 & .886 \\
7. Psicopedagogo & -7.19 & .000 & -3.12 & .002 & -0.78 & .437 \\
8. Empresarial-administración-comercial & -1.19 & .235 & -0.93 & .352 & -0.61 & .542 \\
9. Informática & -12.72 & .000 & -2.46 & .014 & -1.46 & .143 \\
10. Agrario-agropecuario-ambiental & -0.88 & .381 & -1.37 & .170 & -5.91 & .555 \\
11. Artístico plástico-artesanía-moda & -7.68 & .000 & -3.11 & .002 & -2.33 & .020 \\
12. Artístico musical-espectáculo & -6.49 & .000 & -2.99 & .003 & -1.13 & .258 \\
13. Fuerzas armadas-seguridad-protección & -7.94 & .000 & -3.93 & .000 & -0.41 & .682 \\
14. Deportivo & -7.38 & .000 & -3.38 & .001 & -0.03 & .978 \\
15. Turismo-hotelería & -8.03 & .000 & -2.00 & .045 & -0.34 & .738 \\
\hline
\end{tabular}

Nota: Las comparaciones se realizaron con la prueba $U$ de Mann Whitney. 
variable género, aunque solo en diez de los quince campos profesionales con valores que van desde un $\mathrm{p}=.000$ (área deportivo) hasta un $\mathrm{p}=.046$ (área científico); las áreas que no presentan diferencias significativas son: técnico ( $\mathrm{p}=.174)$, empresarial, administración y comercial $(\mathrm{p}=.151)$, informática $(\mathrm{p}=$ $.241)$, agrario-agropecuario y ambiental $(\mathrm{p}=.068)$ y turismo-hotelería $(\mathrm{p}=.072)$.

\section{Grado de estudio}

Tratándose del grado de estudio, se confirma la tendencia observada en el área profesión dado que en la totalidad de campos profesionales no existen diferencias significativas por razón del grado de estudio en los intereses de los jóvenes, sean de cuarto o quinto de secundaria, con valores que van desde un $\mathrm{p}=.067$ (área científico) hasta un $\mathrm{p}=$ .983 (área informática).

\section{Tabla 9}

\section{Comparación de la actividad según género, gestión y grado}

\begin{tabular}{|c|c|c|c|c|c|c|}
\hline \multirow{3}{*}{ Áreas } & \multicolumn{6}{|c|}{ Actividad } \\
\hline & \multicolumn{2}{|c|}{ Género } & \multicolumn{2}{|c|}{ Gestión } & \multicolumn{2}{|c|}{ Grado } \\
\hline & $\mathbf{z}$ & $\mathbf{p}$ & $\mathbf{z}$ & $\mathbf{P}$ & $\mathbf{z}$ & p \\
\hline 1. Científico & -0.05 & .959 & -1.99 & .046 & -1.83 & .067 \\
\hline 2. Técnico & -9.76 & .000 & -1.36 & .174 & -0.85 & .394 \\
\hline 3. Sanitario & -8.36 & .000 & -2.32 & .020 & -0.77 & .444 \\
\hline 4. Científico social-humanidades & -7.51 & .000 & -3.29 & .001 & -0.21 & .836 \\
\hline 5. Jurídico-social & -2.24 & .025 & -1.95 & .051 & -0.93 & .925 \\
\hline 6. Comunicación-información & -2.04 & .041 & -2.39 & .017 & -0.64 & .520 \\
\hline 7. Psicopedagogico & -0.72 & .000 & -2.56 & .011 & -0.58 & .561 \\
\hline 8. Empresarial-administración-Comercial & -2.54 & .011 & -1.44 & .151 & -0.15 & .884 \\
\hline 9. Informática & -7.69 & .000 & -1.17 & .241 & -0.02 & .983 \\
\hline 10. Agrario-agropecuario-ambiental & -2.52 & .012 & -1.82 & .068 & -0.48 & .631 \\
\hline 11. Artístico plástico-artesanía-moda & -7.20 & .000 & -2.91 & .004 & -1.70 & .090 \\
\hline 12. Artístico musical-espectáculo & -7.30 & .000 & -2.31 & .021 & -0.96 & .338 \\
\hline 13. Fuerzas armadas-Seguridad-protección & -7.87 & .000 & -3.43 & .001 & -1.36 & .173 \\
\hline 14. Deportivo & -2.51 & .012 & -4.42 & .000 & -0.19 & .847 \\
\hline 15. Turismo-hotelería & -8.88 & .000 & -1.80 & .072 & -0.38 & .702 \\
\hline
\end{tabular}

Nota: Las comparaciones se realizaron con la prueba $U$ de Mann Whitney. 


\section{SEMEJANZAS Y DIFERENCIAS EN INTERESES}

En las tablas 10 y 11 se identifican los promedios obtenidos por la muestra de estudio en cada uno de los campos profesionales, tomando en cuenta las variables género, gestión y grado de estudio.

Dado que cada campo profesional tiene diferentes puntajes máximos en función de la cantidad de ítems que abarca, se determinó establecer un factor de equivalencia que permitiese comparar el grado de interés, por ello se calcularon porcentajes de logro de interés que nos permitió establecer comparaciones.

\section{Área profesión}

En función de esta área podemos señalar que el campo profesional donde los jóvenes presentan mayor interés es en el artístico musical-espectáculo, lo cual significa que los alumnos están interesados en carreras relacionadas con el canto, la actuación, la música y el baile, tendencia que se refleja en los resultados según las variables de estudio: las mujeres tienen un mayor interés $(47,2 \%)$ que los hombres $(37,6 \%)$, los alumnos de los colegios estatales en mayor medida $(43,2 \%)$ que los de colegios particulares $(37,0 \%)$, y tratándose del grado de estudio, los alumnos del cuarto grado presentan un $43,2 \%$ frente al $41,5 \%$ del quinto grado.

Un segundo campo profesional está referido al empresarial-administración y comercial y está relacionado con ca- rreras como Economista, Administración y Contabilidad. Tratándose de las variables del estudio las diferencias entre los diversos grupos es mínima, tal como se puede identificar: hombres $(41,5 \%)$ y mujeres $(40,4 \%)$, estatal $(41,4 \%$ ) y particular (39,2\%); finalmente, un $41,7 \%$ para los de quinto grado frente al $40,7 \%$ de los de cuarto grado.

En lo que se refiere a la misma área de profesión, entre los campos profesionales de menor interés tenemos el científico social-humanidades, que involucra carreras como la Historia, la Sociología, la Filosofía, el Trabajo Social, la Arqueología, la Antropología y el Arte (pintura, escultura, etcétera). Esta baja de interés es significativa en los colegios particulares con un 16,0 \% frente al 21,8 \% de los colegios estatales. Tratándose del grado, los del quinto obtienen un $21,2 \%$ de interés frente al $21,0 \%$ de los del cuarto grado; finalmente, los hombres obtienen un $21,0 \%$ frente al $21,1 \%$ de las mujeres. Un segundo campo profesional es el psicopedagógico, que se refiere a carreras como las de Educación (especial, inicial, primaria, secundaria y educación física); Psicología Escolar y Educador Social. Las diferencias por variables se encuentran en el género, ya que los varones obtienen un $17,5 \%$ con relación al 27,8 \% de las mujeres; un $17,1 \%$ en centros particulares y un $23,5 \%$ en los estatales, así como un $23,2 \%$ obtenido por los alumnos de quinto grado y $22,2 \%$ por los de cuarto grado. 


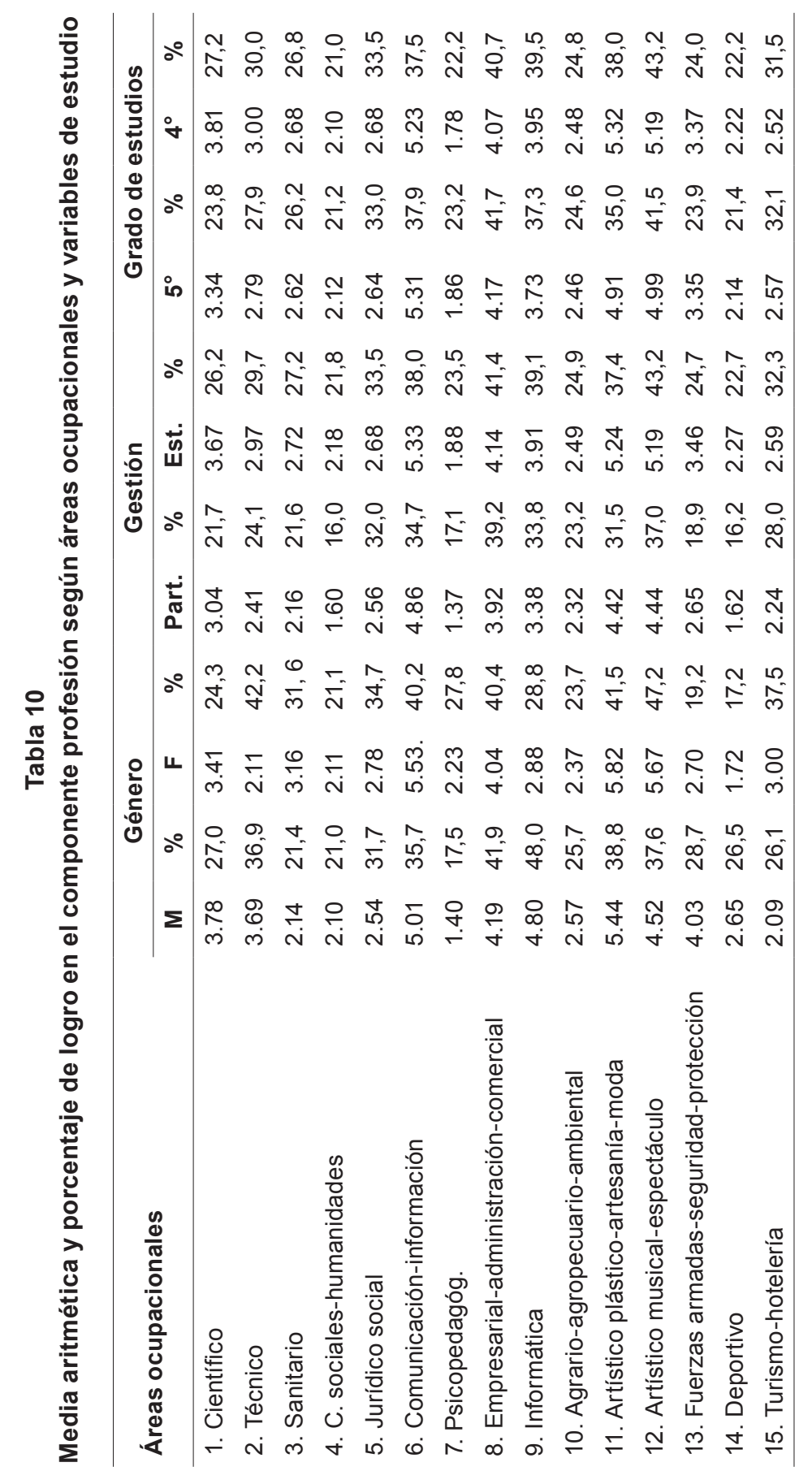


En lo que respecta a los campos profesionales de mayor interés y en función a las variables de estudio, los hombres presentan mayor interés por la informática con $48 \%$ y las mujeres por el campo artístico musical-espectáculo con 47,2 \%; en cuanto al tipo de gestión, los alumnos de colegio particular obtuvieron un 39,2 \%; en el campo profesional, empresarial-administración y comercial, y los de gestión estatal en el campo profesional artístico musicalespectáculo con 43,2\%. Por último, tratándose del grado de estudio, los alumnos del quinto grado obtuvieron un 41,7 $\%$ en el campo profesional empresarial administración-comercial y los de cuarto grado, un 43,2 \% en el campo profesional artístico musical-espectáculo.

En lo relativo a las menores preferencias de interés, por variables, vemos que los hombres muestran muy poco interés en las carreras relacionadas con el campo profesional psicopedagógico, con $17,5 \%$; en cambio, las mujeres muestran desinterés por el campo profesional deportivo, con 17,2 \%; en cuanto a la gestión, los alumnos de colegio particular muestran poco interés por los campos profesionales de las ciencias sociales-humanidades $(16,0 \%)$ y deportivo $(16,2 \%)$ y los de estatal por el campo profesional de ciencias socialeshumanidades $(21,8 \%)$. Por último, tratándose del grado de estudios, tanto los del quinto como los de cuarto grado de secundaria muestran poco interés con relación al campo profesional de cien- cias sociales-humanidades con $21,2 \%$ y $21,0 \%$, respectivamente.

\section{Área actividad}

La complementariedad entre las áreas de profesión y actividad le da consistencia a la intencionalidad de la prueba adaptada; quiere decir que no basta con tener interés por una carrera o profesión sino que también hay que conocer las diversas actividades que implican cada una de ellas. En este contexto, observando los datos de la tabla 11, es importante resaltar que el interés por las actividades está asociado de manera resaltante con el campo profesional artístico musical-espectáculo, y que coincide de manera directa con el interés por las profesiones del mismo grupo, lo cual debe entenderse como un interés consolidado. Tratándose de las variables del estudio, el grupo de mujeres alcanza un $54,1 \%$ frente al 43,1\% de los hombres; los del grupo estatal alcanzan el 49,1\% y los del particular 43,8 \%. En cuanto al grado de estudio, el cuarto grado obtiene $49,1 \%$ y el quinto, $47,7 \%$.

Otro de los campos profesionales donde los alumnos demuestran un mayor conocimiento por las actividades es el de comunicación-información, que está relacionado con carreras como Periodismo, Comunicación y Técnica en Sonido o Imagen, aunque en el área profesión no aparezcan como carreras de interés. En cuanto al género, las mujeres obtienen un porcentaje mayor 


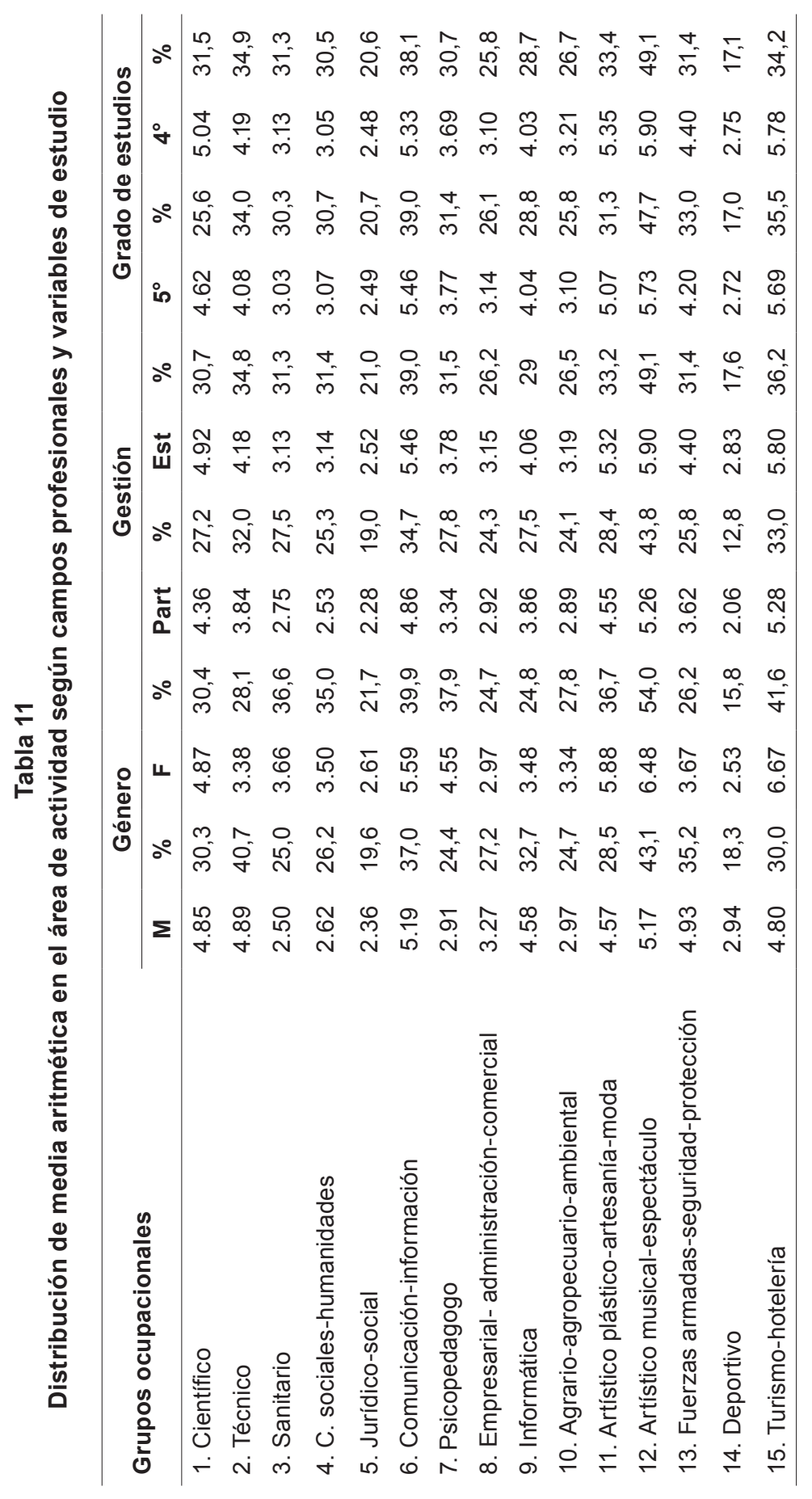


que los hombres: $39,9 \%$ frente al $37,0 \%$, respectivamente. Tratándose de la gestión, los colegios estatales obtienen $39,0 \%$ y los particulares $34,7 \%$. Por último, el quinto grado obtiene un $39,0 \%$ y $38,1 \%$ el cuarto grado.

En contraposición, el poco interés y conocimiento de las actividades se observa en el campo profesional deportivo. Comparativamente las mujeres obtienen solo un $15,8 \%$ con relación al 18,3\% de los hombres; los colegios particulares un $12,8 \%$ y los estatales $17,6 \%$ y los de quinto y cuarto grado alcanzan porcentajes casi idénticos, con 17,1 \% y 17,0\%, respectivamente.

\section{DisCUSIÓN DE RESULTADOS}

Asumiendo lo señalado por Muñiz \& Hambleton (1996), quienes expresan que los test no son instrumentos automáticamente utilizables, deben adaptarse en función de las diferencias interculturales, así como considerando lo señalado por la Comisión Internacional de Test (International Tests Commission, ITC), se realizó la adaptación del IPP-R tomando en consideración las características de los jóvenes de nuestra realidad sociocultural, diferente del contexto de origen donde se elaboró la prueba (España), la cual se convalidó utilizando el criterio de jueces y una prueba piloto, lo que validó fácticamente la adaptación propuesta.

La versión final, aplicada a una muestra de 1567 sujetos, arrojó estadísticamente índices de confiabilidad y validez que nos permiten afirmar que la prueba es válida para una población con las mismas características de la que fue evaluada, lo cual nos permite afirmar que cuando una prueba es adaptada en los marcos metodológicos establecidos y con un procesamiento estadístico pertinente, las consecuencias serán siempre contar con instrumentos funcionales a nuestra realidad que nos permita obtener datos que luego serán utilizados para la toma de decisiones con seres humanos, con un mínimo error.

Los niveles de confiabilidad de la versión adaptada, para cada uno de los campos profesionales, varían entre las categorías moderada y alta. Estos resultados difieren de los niveles de fiabilidad obtenidos en la prueba original para cada uno de los campos que componen la prueba. Los niveles de la versión original resultaron altos en todos los casos. No obstante, a nuestro juicio, es probable que en la versión original los resultados aparezcan sesgados en la medida en que, para el análisis de la fiabilidad, no se han separado las áreas de profesión y actividad.

Es importante mencionar respecto de la confiabilidad que, en la versión adaptada, el alfa de Cronbach es superior en el área profesión con relación a la de actividad en algunos campos profesionales, lo cual se puede explicar por el manejo de información diferencial por parte de los alumnos: conocen profesiones pero no conocen sus actividades básicas, y viceversa. 
En el caso de la validez, en la versión original se consideran dos criterios: validez aparente y validez de contenido, los cuales son analizados desde una perspectiva cualitativa. Bajo este mismo criterio, la versión adaptada también tiene validez aparente y de contenido. En este trabajo se ha considerado, además, la validez de constructo, la cual, en función de los estadísticos aplicados, demuestra que el modelo factorial utilizado ha sido adecuado.

Por otro lado, es importante mencionar que los resultados obtenidos, tal como lo señalan Montero (2005), Tyler (1960) y Super (1964) han permitido corroborar que los intereses relacionados con la elección profesional suelen variar en función del género y del nivel socioeconómico, lo cual implicó la elaboración de baremos diferenciados, expresados en percentiles para varones y mujeres, así como para el tipo de gestión del centro educativo (estatal y particular) al que pertenecían los sujetos evaluados. Complementariamente, se elaboraron baremos por grado de estudios.

En cuanto al interés, teóricamente entendido, tal como lo han señalado Kuder, Super, Wolfman, Gati y Allport, entre otros, enfatizan que es un estado de ánimo que traduce una atención selectiva asociada a la satisfacción, y que se aprende en una relación con el medioambiente. Si se trata del interés vocacional, este se puede entender como una tendencia a preferir en- tre ciertas ocupaciones o profesiones, buscando encontrar satisfacción en su elección. Roe (1956) planteó que los intereses vocacionales suelen afianzarse hacia los 15 años, edad a partir de la cual estos suelen mantenerse estables, información corroborada y sustentada en que no existen diferencias en función del grado, asumiendo los quince años como edad representativa del cuarto grado.

Este parámetro conceptual y teórico se debe tomar en cuenta sobre todo cuando es el medioambiente un factor condicionante asociado al desarrollo de los intereses; por ello, cuando encontramos que la muestra de estudio denota bajos niveles de interés en la mayoría de los campos profesionales, cabe plantearse algunas interrogantes, ¿por qué los jóvenes del cuarto y quinto de secundaria, tanto de colegios públicos como privados, presentan bajo interés por las profesiones?, ¿qué rol juega la escuela en la determinación de los intereses?, ¿qué trabajos desarrollan los profesores o tutores con los alumnos en términos vocacionales?, ¿la familia ayuda u obstaculiza la generación de intereses en sus hijos?

Estas preguntas tienen sentido cuando descubrimos que la tendencia general de elección de los sujetos se orienta a los campos profesionales artístico musical-espectáculo y el empresarialadministración-comercial, con carreras asociadas a la música, el canto, el baile y la actuación, así como a la Economía, 
la Administración o la Contabilidad, mostrando poco interés por las carreras de historiador, sociólogo, filósofo, trabajador social, antropólogo, profesor o psicólogo escolar.

La poca o insuficiente información que reciben los jóvenes en la escuela, el hogar o el medioambiente en general, sobre las profesiones u ocupaciones que tienen presencia en el contexto laboral, determina un escaso interés por estas. Es notoria la poca importancia que le dan los alumnos a las carreras tradicionalmente preferidas por los jóvenes, como las de Medicina, Derecho o las del campo militar, lo cual se traduciría en la ausencia real de alternativas para los jóvenes, que les permita su desarrollo profesional.

La tendencia descrita se confirma cuando se realizan comparaciones en función de las variables género, gestión y grado de estudio, aunque un dato rescatable se relaciona con la presencia marcada de diferencias de elección por género, en menor proporción cuando se trata del tipo de gestión (particularestatal) y casi nula en el caso del grado, lo cual permitiría afirmar que desde el cuarto grado de secundaria los jóvenes ya consolidan su interés. Por lo tanto, no sería necesario esperar al quinto de secundaria para tomar decisiones vocacionales, sino que podrían hacerlo mucho antes, porque ya tendrían un interés consolidado.
En cuanto al área de actividad, el interés se consolida cuando el joven elige una carrera y conoce el conjunto de actividades que ella implica y que la complementariedad solo se ha encontrado en el caso del campo profesional artístico musical-espectáculo, a pesar de que son quince los campos profesionales. Incluso aparece el campo profesional comunicación-información, en el cual se identifica un interés por actividades que conciernen a dicho campo, pero los sujetos desconocen dentro de qué carrera se ubican estas.

\section{ReFERENCIAS}

Álvarez. J. et al. (1998). Guía de desarrollo y orientación profesional. Cuaderno del tutor. Valencia: Promolibro.

Anastasi, S. (1998). Test psicológicos. México, D. F.: Prentice Hall.

Boada, A., \& Di Alessio, M. (2011). Sistema diagnóstico aptitudinal-vocacional automatizado de orientación para aspirantes a ingresar a nivel superior. Experiencia Unefa. Revista Investigaciones Operacionales, 32, 290-302.

Chapman, E. (1991). Orientación vocacional: La elección acertada de carrera. México, D. F.: Trillas.

Contreras, L. (2000). Validación del inventario "Control de Intereses Profesionales» (CIP) de Fernando 
Gutiérrez, en una muestra de jóvenes asistentes a pre-universitario en la Región Metropolitana. Santiago, Chile: Universidad de Chile. (Memoria para optar el título de Psicólogo).

Cortada, N. (1997). El profesor y la orientación vocacional. México, D. F.: Trillas.

Crites, J. (1969). Vocational psychology. The study of vocational behavior and development. Nueva York: McGraw-Hill.

Crites, J. (1974). Psicología vocacional. Buenos Aires: Paidós.

De la Cruz, M. (2010). Intereses y preferencias profesionales. (3. ${ }^{\mathrm{a}} \mathrm{ed}$.). Madrid: TEA Ediciones.

Fernández, M., Cayssials, A., \& Pérez, M. (2009). Curso básico de psicometría. Teoría clásica. Buenos Aires: Lugar Editorial.

Gavilán, M. (2006). La transformación de la orientación vocacional. Hacia un nuevo paradigma. Buenos Aires: Homo Sapiens Ediciones.

Gordillo, M. (1993). Manual de orientación educativa. Madrid: Alianza.

Hernández, G. (2001). Psicología y desarrollo profesional. México, D. F.: Cecsa.

Hernández, R., Fernández-Collado, C., \& Baptista, P. (2010). Metodología de la investigación (5. ${ }^{\mathrm{a}}$ ed.). México, D. F.: McGraw-Hill Interamericana.

Holland, J. (1965). La elección vocacional. Teoría de las carreras. México, D. F.: Trillas.

López, F., \& Figueroa, C. (2002). Persona y profesión: procedimientos y técnicas de selección y orientación. Madrid: TEA.

Muñiz, J., \& Hambleton, R. (1996). Directrices para la traducción y adaptación de los test. Papeles del Psicólogo, 66. Recuperado de http://www. papelesdelpsicologo.es/vernumero. asp?id $=737$

Muñiz, J. (1994). Traducción y adaptación de los test. Papeles del Psicólogo, 59. Recuperado de http://www. papelesdelpsicologo.es/vernumero. asp?id $=626$

Nunally, J. C., \& Bernstein, I. H. (1995). Teoría psicométrica. Nueva York: McGraw-Hill.

Osipow, S. (1991). Teorías sobre la elección de carreras. México, D. F.: Trillas.

Pacheco, J. (2008). Cómo se manifiesta la resistencia de los docentes al uso de las nuevas tecnologías de la información y la comunicación como apoyo en los procesos de enseñanza. Chihuahua: Centro Chihuahuense de Estudios de Posgrado. (Tesis de maestría en desarrollo educativo). 
Recuperado de http://www.cchep. edu.mx/docspdf/cc/056.pdf

Pérez, E., \& Cupani, M. (2006). Desarrollo y validación de un inventario de intereses vocacionales: El CIP-4. Psicothema, 18(2), 238-242.

Pérez, E., \& Fogliato, H. (2004). Desarrollo de un sistema de orientación vocacional asistido por computadora: el SOVI 3. Revista Iberoamericana de Diagnóstico y Evaluación Psicológica, 17(1), 9-26.
Rivas, F. (1995). Manual de asesoramiento y orientación vocacional. Madrid: Síntesis.

Shaughnessy, J., Zechmeister, E., \& Zechmeister, J. (2007). Métodos de investigación en psicología (7. ${ }^{\mathrm{a}}$ ed.). México: McGraw-Hill Interamericana.

Super, D. (1967). Psicología de los intereses y las vocaciones. Buenos Aires: Kapelusz.

Valdés, V. (2006). Vocación y profesión. México, D. F.: Pearson. 